\title{
INVARIANT RELATIONS FOR THE BOWEN-SERIES TRANSFORM
}

\author{
VINCENT PIT
}

\begin{abstract}
Consider the Bowen-Series transform $T$ associated with an even corners fundamental domain of finite volume for some Fuchsian group $\Gamma$. We prove a generic invariance result that abstracts Series' orbit-equivalence theorem to families of relations on the unit circle. Two applications of this result are developed. We first prove that $T$ satisfies a strong-orbit equivalence property, which allows to identify its hyperbolic periodic orbits with primitive hyperbolic conjugacy classes of $\Gamma$. Then, we show thanks to the invariance theorem that the eigendistributions for the eigenvalue 1 of the transfer operator of $T$ with spectral parameter $s \in \mathbb{C}$ are in bijection with smooth bounded eigenfunctions for the eigenvalue $s(1-s)$ of the hyperbolic Laplacian on the quotient $\mathbb{D} / \Gamma$.
\end{abstract}

\section{INTRODUCTION}

Let $\mathbb{D}$ be the Poincaré disk model for the hyperbolic space of constant curvature -1 , and let $\Gamma$ be a Fuchsian group of finite covolume that admits a fundamental domain $\mathcal{D}$ that is not a compact triangle. In the classical article [BS79], Bowen and Series associated a transformation $T$ mapping the unit circle $\mathbb{S}^{1}$ to itself (where $\mathbb{S}^{1}$ is seen as the boundary at the infinity of the hyperbolic space) with any such fundamental domain that satisfies the technical even corners property. This so-called Bowen-Series transform has three important properties: it naturally preserves a finite Markov partition of the circle in intervals $\left(I_{k}\right)$, it acts in a piecewise manner by a finite family of generators of $\Gamma$, and it is orbit-equivalent to the Fuchsian group $\Gamma$.

Theorem 1.1 (Series). Let $x, y \in \mathbb{S}^{1}$. Then

$$
\exists g \in \Gamma, y=g(x) \Leftrightarrow \exists p, q \geq 0, T^{p}(x)=T^{q}(y) .
$$

This article aims to revisit this property and its consequences in the setting of finite covolume Fuchsian groups.

The orbit-equivalence property translates the combinatorics given by the Markov partition preserved by $T$ to relations between points of $\mathbb{S}^{1}$. We will prove that the arguments leading to this property can be extended to prove that families of relations of $\mathbb{S}^{1}$ that satisfy some regularity and consistency conditions and that are invariant under $T$ are actually invariant under the whole group $\Gamma$. With a suitable choice for the family of relations, this will show, in particular, that $T$ satisfies a strong orbit-equivalence property, in that the exponents $p$ and $q$ that appear in the orbit-equivalence theorem can be chosen to be locally constant.

Received by the editors December 7, 2011.

2010 Mathematics Subject Classification. Primary 37D40; Secondary 37C30, 58C40. 
Theorem 4.2. Let $\gamma_{k}[x]$ be the isometry by which acts the $k$-th iterate of the Bowen-Series transform in a neighbourhood of $x$. For every $x \in \mathbb{S}^{1}$ and $g \in \Gamma$, there exist $p, q \geq 0$ such that

$$
\gamma_{p}[x]=\gamma_{q}[g(x)] g .
$$

This theorem will allow us to completely describe the periodic orbits of $T$ by mapping them exactly to conjugacy classes of isometries of $\Gamma$.

Theorem 5.3. There is a bijection between:

- the hyperbolic periodic orbits of $T$,

- the conjugacy classes of primitive hyperbolic isometries of $\Gamma$.

The accuracy of the invariance theorem will ensure that we have a precise correspondence where all hyperbolic isometries appear as periodic orbits of $T$.

The invariance theorem has a seemingly unrelated application to the spectral theory of the transfer operator associated with the Bowen-Series transform $T$. For any parameter $s \in \mathbb{C}$, it is a linear operator that acts on the space $E$ of functions mapping $\mathbb{S}^{1}$ to $\mathbb{C}$ whose restriction to every interval $I_{k}$ of the Markov partition preserved by $T$ is continuously differentiable on some open neighbourhood of the closure of $I_{k}$. It is given by

$$
\mathcal{L}_{s}: \phi \in E \mapsto\left(y \mapsto \sum_{T(x)=y} \frac{\phi(x)}{\left|T^{\prime}(x)\right|^{s}}\right) .
$$

Generalizing a result of Pollicott from Pol91, we will show that the eigendistributions of $\mathcal{L}_{s}$ that are smooth enough are exactly the Helgason boundary values of eigenfunctions of the Laplacian on the finite volume quotient $\mathbb{D} / \Gamma$.

Theorem 6.6. For every $s \in \mathbb{C}$ such that $0<\Re(s) \leq 1$, there is an isomorphism between:

- the space of linear functionals $\nu$ that can be written as the weak derivative of a $\Re(s)$-Hölder function and that satisfy

$$
\forall \phi \in E,\left\langle\nu, \mathcal{L}_{s} \phi\right\rangle=\langle\nu, \phi\rangle,
$$

- the space of smooth bounded $\Gamma$-invariant eigenfunctions of the hyperbolic Laplacian in $\mathbb{D}$ for the eigenvalue $s(1-s)$.

It is given by $\nu \mapsto\left(z \mapsto\left\langle\nu, P^{s}(z,).\right\rangle\right)$ where $P^{s}(z, x)$ is the Poisson kernel.

\section{Preliminaries}

Let $\Gamma$ be a Fuchsian group of finite covolume, and $\mathcal{D}$ a (finite volume) fundamental domain for its action on the hyperbolic space $\mathbb{D}$.

We will denote by $S$ the set of its sides and by $V$ the set of its vertices in $\overline{\mathbb{D}}=\mathbb{D} \cup \partial \mathbb{D}$. We will always assume that $\mathcal{D}$ is a geodesically convex polygon. In that case, since $\Gamma$ is geometrically finite, $n=\operatorname{card} S=$ card $V$ is finite. Every side $s \in S$ can be paired with another side $t \in S$ by some isometry $\gamma_{s}$ such that $\gamma_{s}(s)=t, \gamma_{t}(t)=s$ and $\gamma_{s}=\gamma_{t}^{-1}$. Note that a side is allowed to be paired with itself, in which case $\gamma_{s}$ is an elliptic isometry of $\Gamma$ of order 2 whose center is the midpoint of the side $s$. Since $\mathcal{D}$ is convex, $\left\{\gamma_{s} \mid s \in S\right\}$ generates $\Gamma$.

Pick $v \in V$. There are exactly two sides $l_{v}, r_{v} \in S$ that contain $v$, where $l_{v}$ (respectively, $r_{v}$ ) is the left side (respectively, right side) of the fundamental domain 
at $v$. Put differently, the angle of the corner subtended by the fundamental domain at $v$ and going from $r_{v}$ to $l_{v}$ is non-negative. $\gamma_{r_{v}}$ will be commonly shortened as $\gamma_{v}$.

There is exactly one other vertex $l(v) \in V$ (respectively, $r(v)$ ) that lies on $l_{v}$ (respectively, $r_{v}$ ) and is different from $v$. Since the left (respectively, right) side at $v$ is the right (respectively, left) side as $l(v)$ (respectively, $r(v)$ ), we have that

$$
r_{l(v)}=l_{v} \quad \text { and } \quad l_{r(v)}=r_{v} .
$$

This implies that the two applications $r: V \rightarrow V$ and $l: V \rightarrow V$ are permutations of $V$ that are inverse of each other, i.e. $l=r^{-1}$. Moreover, $\gamma_{l_{v}}$ and $\gamma_{v}$ can be expressed as

$$
\gamma_{l_{v}}=\gamma_{l(v)} \quad \text { and } \quad \gamma_{r_{v}}=\gamma_{v}=\gamma_{r(v)} .
$$

Since $\gamma_{v}$ sends the endpoints of $r_{v}$ and $l_{v}$ onto endpoints of other sides of the fundamental domain, the applications

$$
\begin{aligned}
\sigma: V & \rightarrow V \\
v & \mapsto \gamma_{v}(v)
\end{aligned} \quad \text { and } \quad \begin{aligned}
\tau: V & \rightarrow V \\
&
\end{aligned} \quad \begin{aligned}
v & \mapsto \gamma_{l(v)}(v)
\end{aligned}
$$

are well defined. Using the fact that $\gamma_{v}$ and $\gamma_{l_{v}}$ are conformal and cannot map $\mathcal{D}$ onto itself, it is easy to see that $\gamma_{v}\left(r_{v}\right)$ and $\gamma_{l_{v}}\left(l_{v}\right)$, which are sides of $\mathcal{D}$ that respectively go through $\sigma(v)$ and $\tau(v)$, must be given by

$$
\gamma_{v}\left(r_{v}\right)=l_{\sigma(v)} \quad \text { and } \quad \gamma_{l_{v}}\left(l_{v}\right)=r_{\tau(v)} .
$$

Thanks to inverse relation for side-pairing isometries, this means that

$$
\gamma_{v}^{-1}=\gamma_{l \sigma(v)} \quad \text { and } \quad \gamma_{l_{v}}^{-1}=\gamma_{r \tau(v)} .
$$

Specializing the relation $\gamma_{l \sigma(v)} \gamma_{v}=$ id on the vertex $v$, we get that $\sigma$ and $\tau$ are permutations of $V$ that are inverse of each other, i.e. $\sigma^{-1}=\tau$. Moreover, equation (11) restricted to the vertex different from $v$ gives that

$$
\tau r=l \sigma .
$$

$\sigma$ being a permutation, we call $p_{v}$ the order of the vertex $v \in V$ under the action of $\sigma$. If $v$ is an inner vertex (that is, $v \in V \cap \mathbb{D}$ ), we denote by $q_{v}$ the order of the stabilizer of $v$ in $\Gamma . q_{v}>1$ if and only if $\Gamma$ contains a primitive (i.e. that are not a non-trivial power of another isometry) elliptic isometry of center $v$ and order $q_{v}$.

We have already seen that the $\left(\gamma_{v}\right)_{v \in V}$ generates $\Gamma$. As for the relations of the group, they are encoded in the $\sigma$-orbits:

Proposition 2.1. For every $v \in V \cap \mathbb{D}$,

$$
\left(\gamma_{\sigma^{p_{v}-1}(v)} \cdots \gamma_{v}\right)^{q_{v}}=i d .
$$

Proof. $C=\left\{v, \ldots, \sigma^{p_{v}-1}(v)\right\}$ is a cycle of length $p_{v}$ on the boundary of $\mathcal{D}$. If $\theta$ is the sum of the angles subtended by $\mathcal{D}$ at each point of $C$, theorem 9.3 .5 of [Bea83] shows that $q_{v} \theta=2 \pi$. However, $\gamma=\gamma_{\sigma^{p_{v}-1}(v)} \cdots \gamma_{v}$ fixes $v$ and hence acts as a rotation of center $v$ and of angle $\theta$. This means that $\gamma$ is of order $q_{v}$.

For every $v \in V \cap \mathbb{D}$, define

$$
N_{v}=\{g \text { geometrical geodesics } \mid v \in g \text { and } \exists \gamma \in \Gamma, \gamma(g) \in S\}
$$

and let $n_{v}=$ card $N_{v}$ and $m_{v}=2 n_{v}$. Since $g \in N_{v} \mapsto \gamma_{v}(g) \in N_{\sigma(v)}$ is a bijection, $v \mapsto n_{v}$ is constant on the $\sigma$-orbits. 


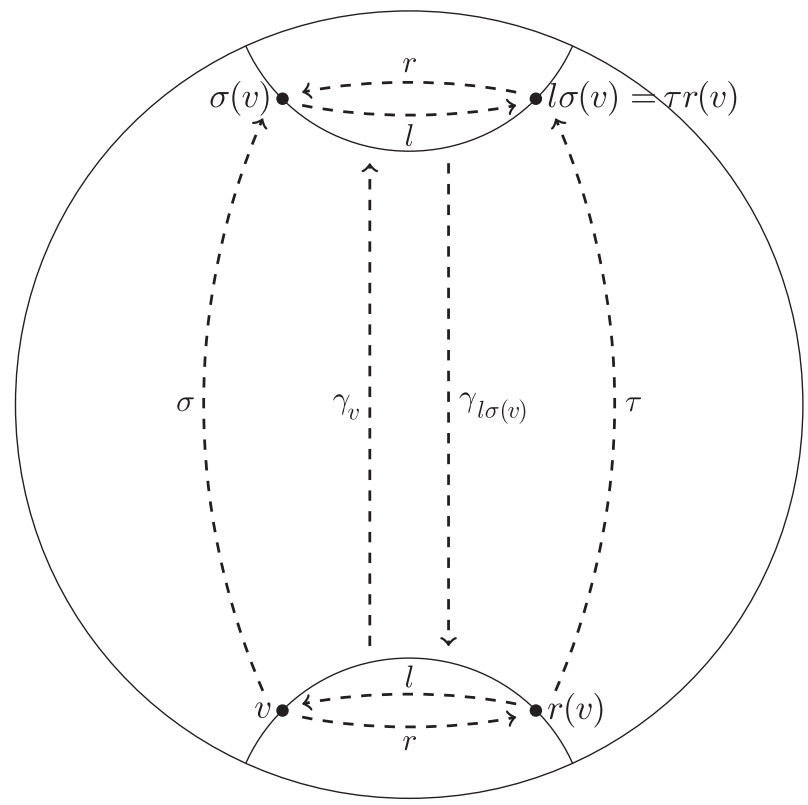

Figure 1. The permutations $\sigma, \tau, l$ and $r$

In order to properly define the Bowen-Series transform, we need to assume an additional condition on the fundamental domain $\mathcal{D}$, called the even corners condition: we require that no geodesic of any $N_{v}$ crosses the interior of $\mathcal{D}$. Note that there are relatively few fundamental domains that satisfy this condition: theorem 9.4.5 from Bea83 implies that for $p \in \mathbb{D}$ outside from a set of measure 0 , the Dirichlet domain of $\Gamma$ based at $p$ has exceptional cycles of length 3 , which forbids it from being even corners. However, given the signature of a Fuchsian group, it is possible to find a group with this signature and a fundamental domain for this group that satisfies the even corners condition. This construction is developed in BS79.

We label $\left(a_{v}^{0}, \ldots, a_{v}^{m_{v}-1}\right)$ the endpoints in $\mathbb{S}^{1}$ of the geodesics of $N_{v}$, numbered anticlockwise starting from the endpoint of $l_{v}$ directed from $v$ towards $l(v)$. With this notation, $l_{v}$ (respectively, $r_{v}$ ) directed from $v$ to $l(v)$ (respectively, $r(v)$ ) is parameterized by $\left(a_{v}^{n_{v}}, a_{v}^{0}\right)$ (respectively, $\left.\left(a_{v}^{n_{v}-1}, a_{v}^{m_{v}-1}\right)\right)$. Thanks to the even corners condition, we know that the triangle $\left(a_{v}^{n_{v}-1}, v, a_{v}^{n_{v}}\right)$ is vertically opposite to the fundamental domain at $v$, which ensures that

$$
a_{v}^{n_{v}-1}=a_{r(v)}^{0} \quad \text { and } \quad a_{v}^{n_{v}}=a_{l(v)}^{m_{l(v)}-1} .
$$

Since $\gamma_{v}$ is conformal, its action on the $a_{v}^{k}$ must preserve their ordering

$$
\gamma_{v}\left(a_{v}^{k}\right)=a_{\sigma(v)}^{k+1} \quad \text { and } \quad \gamma_{v}\left(a_{r(v)}^{k}\right)=a_{l(\sigma(v))}^{k-1} .
$$

When $v$ is a cusp, we artificially set $n_{v}=3$ so that $a_{v}^{1}=a_{v}^{2}=a_{v}^{3}=a_{v}^{4}=v$. Note that in this case the relation $\gamma_{v}\left(a_{v}^{k}\right)=a_{\sigma(v)}^{k+1}$ is not satisfied. With this convention, the reunion $A=\left\{a_{v}^{k} \mid v \in V\right.$ and $\left.k \in \llbracket 0 ; m_{v}-1 \rrbracket\right\}$ of all the endpoints is always a finite set, even in the non-compact case. 


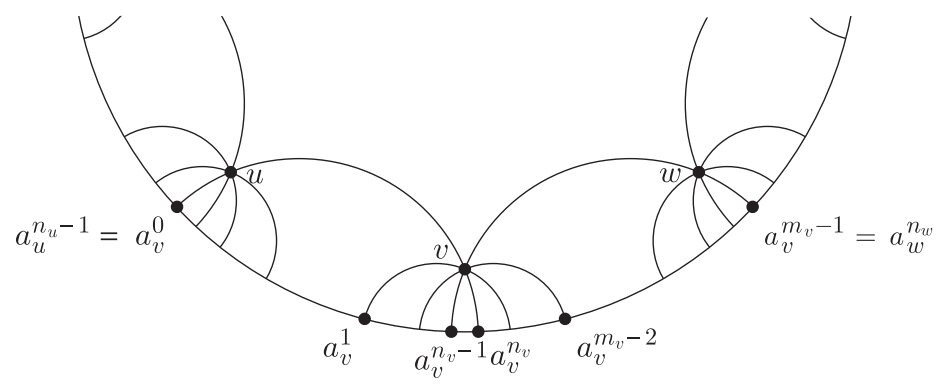

FiguRE 2. Endpoints $a_{v}^{k}$ around an inner vertex $v$

We will also need an additional condition on the fundamental domain to ensure that these endpoints are correctly separated at the infinity.

Proposition 2.2. Suppose that $\mathcal{D}$ is not a compact triangle. Pick $v \in V$ and let $w=r(v)$. Then

$$
\left.\left.a_{w}^{n_{w}-1} \in\right] a_{v}^{m_{v}-2} ; a_{w}^{n_{w}}\right] .
$$

Proof. If $a_{w}^{n_{w}-1} \in\left[a_{v}^{n_{v}-1} ; a_{v}^{m_{v}-2}\right]$ and $n_{w} \geq 3$, then $a_{w}^{1} \in\left[a_{v}^{n_{v}-1} ; a_{v}^{m_{v}-2}[\right.$ and geodesics $\left(v, a_{v}^{m_{v}-2}\right)$ and $\left(w, a_{w}^{1}\right)$ must cross at some $z \in \mathbb{D}$. In that case, the compact triangle $(v, w, z)$ is a copy of a fundamental domain, which is impossible. The only possible alternative is $n_{w}=2$ and $a_{w}^{n_{w}-1}=a_{v}^{m_{v}-2}$, but that would mean that $\mathcal{D}$ is a triangle with a cusp and right angles at $v$ and $w$, which is also impossible.

We can now define the Bowen-Series transforms. Let

$$
\left.\left.I_{v}^{L}=\right] a_{v}^{n_{v}} ; a_{r(v)}^{n_{r(v)}}\right] \quad \text { and } \quad I_{v}^{R}=\left[a_{v}^{n_{v}-1} ; a_{r(v)}^{n_{r(v)}-1}[.\right.
$$

Note that $\left(I_{v}^{L}\right)_{v \in V}$ and $\left(I_{v}^{R}\right)_{v \in V}$ are partitions of $\mathbb{S}^{1}$ in intervals. The left and right Bowen-Series transforms are given by

$$
\begin{aligned}
T_{L}: \quad \mathbb{S}^{1} & \rightarrow \mathbb{S}^{1} \\
x \in I_{v}^{L} & \mapsto \gamma_{v}(x)
\end{aligned} \quad \text { and } \quad T_{R} \quad: \quad \begin{array}{cl}
\mathbb{S}^{1} & \rightarrow \mathbb{S}^{1} \\
x \in I_{v}^{R} & \mapsto \gamma_{v}(x) .
\end{array}
$$

Now, the only endpoints of $A$ that lie in $I_{v}^{L}$ (respectively, $I_{v}^{R}$ ) are of the form $a_{v}^{k}$ or $a_{r(v)}^{k}$, so $A$ is stable under $T_{L}$ (respectively, $T_{R}$ ). This means that the partition $\left(I_{k}^{L}\right)$ (respectively, $\left(I_{k}^{R}\right)$ ) of $\mathbb{S}^{1}$ in intervals half-open on the left (respectively, right) and delimited by the points of $A$ is a Markov partition for $T_{L}$ (respectively, $T_{R}$ )

$$
T_{L}\left(I_{j}^{L}\right) \cap I_{k}^{L} \neq \emptyset \Rightarrow I_{k}^{L} \subset T_{L}\left(I_{j}^{L}\right) \quad \text { and } \quad T_{R}\left(I_{j}^{R}\right) \cap I_{k}^{R} \neq \emptyset \Rightarrow I_{k}^{R} \subset T_{R}\left(I_{j}^{R}\right) .
$$

It is well known that $T_{L}$ and $T_{R}$ are eventually expansive at any point of $\mathbb{S}^{1}$ that is not a cusp of the fundamental domain.

From this point, we will only focus on the right Bowen-Series transform, and $T,\left(I_{v}\right)$ will be used instead of $T_{R},\left(I_{v}^{R}\right)$. By symmetry, all the following results are also true for the left Bowen-Series transform. 


\section{INVARIANCE THEOREM}

Let $\mathbb{I}$ be the set of all right half-open intervals of $\mathbb{S}^{1}$, which is the image of all intervals $\left[a ; b\left[\subset\left[0 ; 2 \pi\left[\right.\right.\right.\right.$ by $t \mapsto \exp (i t)$. Note that $\mathbb{S}^{1} \in \mathbb{I}$ since $\mathbb{S}^{1}=\exp (i[0 ; 2 \pi[)$. The group $\Gamma$ naturally acts on the left on $\mathbb{I}$ by $\gamma([a ; b[)=[\gamma(a) ; \gamma(b)[$.

Let $Z$ be a set on which $\Gamma$ also acts on the left, and $F$ a map from $\mathbb{I} \times Z$ onto $\mathbb{C}$. If $I \in \mathbb{I}$ and $\gamma \in \Gamma$, we say that $F$ satisfies property $\mathcal{I}(I, \gamma)$ when

$$
\forall z \in Z, F(I, z)=F(\gamma(I), \gamma(z)) .
$$

The main theorem of this section states that if $F$ satisfies the finite number of properties $\mathcal{I}\left(I_{v}, \gamma_{v}\right)$ where $\left(I_{v}, \gamma_{v}\right)$ are given by the Bowen-Series coding, as well as some technical conditions, then it satisfies $\mathcal{I}(I, \gamma)$ for every $I \in \mathbb{I}$ and $\gamma \in \Gamma$. This generalizes the orbit-equivalence property to invariance relations for $F$. In the following, we will apply this theorem twice with suitable choices for $Z$ and $F$ : first with $Z=\mathbb{S}^{1} \times \Gamma$ for proving theorem 4.2, and later with $Z$ being the space of continuously differentiable functions on $\mathbb{S}^{1}$ for the proof of theorem 6.6.

Theorem 3.1. Suppose that $F: \mathbb{I} \times Z \rightarrow \mathbb{C}$ satisfies the four following properties:

(i) (Additivity for contiguous intervals): If $I, J \in \mathbb{I}$ are contiguous and disjoint, then $\forall z \in Z, F(I \sqcup J, z)=F(I, z)+F(J, z)$.

(ii) (Continuity): If $\left(b_{n}\right)$ is an increasing sequence of points in $] a ; b[$ that converges towards $b$, then $\forall z \in Z, \lim _{n \rightarrow+\infty} F\left(\left[a ; b_{n}[, z)=F([a ; b[, z)\right.\right.$.

(iii) (Inclusion): For every pair $(I, \gamma)$ such that $F$ satisfies $\mathcal{I}(I, \gamma), F$ also satisfies $\mathcal{I}(J, \gamma)$ for all $J \in \mathbb{I}, J \subset I$.

(iv) (Bowen-Series invariance): $F$ satisfies $\mathcal{I}\left(I_{v}, \gamma_{v}\right)$ for every $v \in V$.

Then $F$ satisfies $\mathcal{I}(I, \gamma)$ for every $(I, \gamma)$.

When $F$ satisfies properties (i), (ii) and (iii), we say that $(F(., z))_{z \in Z}$ is a family of relations. These conditions do not imply that $(F(., z))_{z \in Z}$ is a family of measures because they must satisfy the additivity property only for contiguous intervals and not for generic disjoints intervals.

The sketch of the proof is as follows: the inclusion property ensures that we only need to prove that $F\left(\mathbb{S}^{1}, z\right)=F\left(\mathbb{S}^{1}, \gamma(z)\right)$ for every $z$ and $\gamma$, but since $\left(\gamma_{v}\right)_{v \in V}$ generates $\Gamma$ it is actually sufficient to show that $F\left(\mathbb{S}^{1}, z\right)=F\left(\mathbb{S}^{1}, \gamma_{v}(z)\right)$ for every $z$ and vertex $v$. By the additivity property, one way to get this relation is to find a finite partition $\left(I_{i}\right)$ of $\mathbb{S}^{1}$ such that $\mathcal{I}\left(I_{i}, \gamma_{v}\right)$ is satisfied for every $i$. We then remark that since $\gamma_{l \sigma(v)}=\gamma_{v}^{-1}$,

$$
F\left(\gamma_{l \sigma(v)}\left(I_{l \sigma(v)}\right), \gamma_{l \sigma(v)}(z)\right)=F\left(I_{l \sigma(v)}, z\right)=F\left(\gamma_{v} \gamma_{v}^{-1}\left(I_{l \sigma(v)}\right), \gamma_{v} \gamma_{v}^{-1}(z)\right)
$$

so property $\mathcal{I}\left(I_{l \sigma(v)}, \gamma_{l \sigma(v)}\right)$ implies property $\mathcal{I}\left(\gamma_{l \sigma(v)}\left(I_{l \sigma(v)}\right), \gamma_{v}\right)$. It is then enough to prove that $\mathcal{I}\left(I, \gamma_{v}\right)$ is true for the two intervals obtained when you remove $I_{v}$ and $\gamma_{l \sigma(v)}\left(I_{l \sigma(v)}\right)$ from the circle, which is done by induction thanks to the continuity and inclusion properties, but also to the group relations encoded at every inner vertex of the fundamental domain.

Recall that $I_{v}=\left[a_{v}^{n_{v}-1} ; a_{r(v)}^{n_{r(v)}-1}[\right.$ for the (right) Bowen-Series coding. Let $A_{v}=\left[a_{v}^{n_{v}-1} ; a_{v}^{n_{v}}\left[\right.\right.$ and $B_{v}=\left[a_{v}^{n_{v}-2} ; a_{v}^{n_{v}-1}\left[\right.\right.$, so that $\gamma_{l(v)}\left(A_{v}\right)=B_{\tau(v)}$. Those intervals $A_{v}$ and $B_{v}$ are empty if and only if $v$ is a cusp, and they fill the gaps left by $I_{v}$ and $\gamma_{l \sigma(v)}\left(I_{l \sigma(v)}\right)$ in the sense that

$$
\mathbb{S}^{1}=I_{v} \sqcup \gamma_{l \sigma(v)}\left(I_{l \sigma(v)}\right) \sqcup B_{v} \sqcup A_{r(v)} .
$$


$A_{v}$ is an interval that faces the fundamental domain, to which two natural generators $\gamma_{v}$ and $\gamma_{l(v)}$ are associated. Since $A_{v} \subset I_{v}$, and thanks to the inclusion property, we already know that $F$ satisfies $\mathcal{I}\left(A_{v}, \gamma_{v}\right)$. We will start by proving that $F$ also satisfies $\mathcal{I}\left(A_{v}, \gamma_{l(v)}\right)$ for every $v \in V$.

Let $\nu(v)=r \sigma^{n_{v}-1}(v)$. This is a permutation of $V$ : if $r \sigma^{n_{v}-1}(v)=r \sigma^{n_{w}-1}(w)$, then $v=\sigma^{n_{w}-1-\left(n_{v}-1\right)}(w)$. This implies that $v$ and $w$ are then on the same $\sigma$-orbit, so $n_{v}=n_{w}$ and then $v=w$.

Denote by $g_{v}$ the product $\gamma_{\sigma^{n_{v}-2}(v)} \ldots \gamma_{v}$, so that $g_{v}(v)=l \nu(v)$. When $v$ is not a cusp,

$$
g_{v}\left(a_{v}^{n_{v}}\right)=a_{\sigma^{n_{v}-1}(v)}^{m_{v}-1}=a_{\nu(v)}^{n_{\nu(v)}} \quad \text { and likewise } \quad g_{v}\left(a_{v}^{0}\right)=a_{\nu(v)}^{0} .
$$

Let $\kappa_{v}$ be the smallest nonnegative integer $k$ such that $\nu^{k}(v)$ is a vertex at the infinity of the fundamental domain (or $\infty$ if there is no such $k$ ).

For all $v \in V$, we define by induction a sequence $\left(x_{v}^{p}\right)_{p \geq 0}$ of points of $\mathbb{S}^{1}$ :

- If $v$ is a cusp, then: $\forall p \geq 0, x_{v}^{p}=v$.

- If $v$ is not a cusp, then $x_{v}^{0}=a_{v}^{n_{v}-1}$ and $\forall p \geq 0, x_{v}^{p+1}=g_{v}^{-1}\left(x_{\nu(v)}^{p}\right)$.

Lemma 3.2. If $p \geq \kappa_{v}$, then $x_{v}^{p}=a_{v}^{n_{v}}$.

Proof. Let $p=q+\kappa_{v}$ with $q \geq 0$. In order to make the notation easier to follow, let $\kappa=\kappa_{v}$ and $v_{j}=\nu^{j}(v)$ for every nonnegative integer $j$. Since $v=v_{0}, v_{1}, \ldots, v_{\kappa-1}$ are inner vertices by minimality of $\kappa$, we can apply $\kappa$ times the recurrence relation to $x_{v}^{p}$ and

$$
x_{v}^{p}=x_{v}^{q+\kappa}=g_{v}^{-1}\left(x_{v_{1}}^{q+\kappa-1}\right)=\ldots=g_{v}^{-1} \ldots g_{v_{\kappa-1}}^{-1}\left(x_{v_{\kappa}}^{q}\right) .
$$

However, $v_{\kappa}$ is a cusp, so $x_{v_{\kappa}}^{q}=v_{\kappa}=a_{v_{\kappa}}^{n_{v_{\kappa}}}$ and then $x_{v}^{p}=g_{v}^{-1} \ldots g_{v_{\kappa-1}}^{-1}\left(a_{v_{\kappa}}^{n_{v_{\kappa}}}\right)$. Now recall that $v_{\kappa-1}=\nu^{-1}\left(v_{\kappa}\right)$ is not a cusp, so relation (2) gives

$$
x_{v}^{p}=g_{v}^{-1} \ldots g_{v_{\kappa-2}}^{-1} g_{v_{\kappa-1}}^{-1}\left(a_{\nu\left(v_{\kappa-1}\right)}^{n_{\left.\nu v_{\kappa-1}\right)}}\right)=g_{v}^{-1} \ldots g_{v_{\kappa-2}}^{-1}\left(a_{v_{\kappa-1}}^{n_{v_{\kappa-1}}}\right) \text {. }
$$

By repeating this argument for $v_{\kappa-2}, \ldots, v_{1}$, we finally get that $x_{v}^{p}=a_{v}^{n_{v}}$.

Lemma 3.3. If $1 \leq p<\kappa_{v}$, then $\left.x_{v}^{p} \in\right] x_{v}^{p-1} ; a_{v}^{n_{v}}[$.

Proof. We prove this by induction over $p \geq 1$. First, since $\kappa_{v}>1, v$ and $\nu(v)$ are both inner vertices. Hence, proposition 2.2 gives that

$$
\left.x_{v}^{1}=g_{v}^{-1}\left(a_{\nu(v)}^{n_{\nu(v)}-1}\right) \in g_{v}^{-1}(] a_{l \nu(v)}^{m_{v}-2} ; a_{\nu(v)}^{n_{\nu(v)}}=a_{l \nu(v)}^{m_{v}-1}[)=\right] a_{v}^{n_{v}-1}=x_{v}^{0} ; a_{v}^{n_{v}}[.
$$

Now take $p$ such that $2 \leq p<\kappa_{v}$. Since $1 \leq p-1<\kappa_{v}-1=\kappa_{\nu(v)}$, we get $\left.x_{\nu(v)}^{p-1} \in\right] x_{\nu(v)}^{p-2} ; a_{\nu(v)}^{n_{\nu(v)}}[$ by induction. However, $v$ is not a cusp, so

$$
\left.x_{v}^{p}=g_{v}^{-1}\left(x_{\nu(v)}^{p-1}\right) \in g_{v}^{-1}(] x_{\nu(v)}^{p-2} ; a_{\nu(v)}^{n_{\nu(v)}}=a_{l \nu(v)}^{m_{v}-1}[)=\right] x_{v}^{p-1} ; a_{v}^{n_{v}}[.
$$

Lemma 3.4. $\lim _{p \rightarrow+\infty} x_{v}^{p}=a_{v}^{n_{v}}$.

Proof. If $\kappa_{v}<\infty$, the $x_{v}^{p}$ is stationary at $a_{v}^{n_{v}}$ as soon as $p \geq \kappa_{v}$. In that case, the $\left(x_{v}^{p}\right)_{p \geq 0}$ sequence does converge towards $a_{v}^{n_{v}}$.

Now suppose that $\kappa_{v}=\infty$, i.e. $\nu^{j}(v)$ is not a cusp for every $j \geq 0$. The previous lemma shows then that $\left(x_{v}^{p}\right)_{p \geq 0}$ is an increasing sequence that stays in a non-trivial interval $] a_{v}^{n_{v}-1} ; a_{v}^{n_{v}}\left[\right.$ of $\mathbb{S}^{1}$, hence it must converge to some $\left.\left.\xi_{v} \in\right] a_{v}^{n_{v}-1} ; a_{v}^{n_{v}}\right]$. We only have to prove that $\xi_{v}=a_{v}^{n_{v}}$. 
Since $\nu$ is a permutation of $V$, there exists $r_{v}>0$ minimal such that $\nu^{r_{v}}(v)=v$. Note $G_{v}=g_{\nu^{r_{v}-1}} \ldots g_{\nu(v)} g_{v}$. Thanks to relation (2),$g_{v}\left(a_{v}^{n_{v}}\right)=a_{\nu(v)}^{n_{\nu(v)}}$ so we get by induction $G_{v}\left(a_{v}^{n_{v}}\right)=a_{v}^{n_{v}}$. Likewise, we can also see that $G_{v}\left(a_{v}^{0}\right)=a_{v}^{0}$. And since $g_{v}\left(x_{v}^{p+1}\right)=x_{\nu(v)}^{p}$, we have $g_{v}\left(\xi_{v}\right)=z_{\nu(v)}$ when $p$ goes to infinty; hence $G_{v}\left(\xi_{v}\right)=\xi_{v}$. If $\xi_{v} \neq a_{v}^{n_{v}}, G_{v}$ must fix three distinct points of $\mathbb{S}^{1}$, so it has to be the identity. In that case $G_{v}\left(x_{v}^{0}\right)=x_{v}^{0}=a_{v}^{n_{v}-1}$, but on the other hand $G_{v}\left(x_{v}^{0}\right)=x_{v}^{r_{v}}$ must lie in $] x_{v}^{r_{v}-1} ; a_{v}^{n_{v}}$ [ following lemma 3.3. This is impossible since this interval does not contain $a_{v}^{n_{v}-1}$.

In a similar fashion, we define $\left(y_{\tau(v)}^{p}\right)_{p \geq 0}=\left(\gamma_{l(v)}\left(x_{v}^{p}\right)\right)_{p}=\left(\gamma_{\tau(v)}^{-1}\left(x_{v}^{p}\right)\right)_{p}$ that satisfies:

(a) $y_{v}^{0}=a_{v}^{n_{v}-2}$,

(b) $\forall p \geq \kappa_{v}, y_{v}^{p}=a_{v}^{n_{v}-1}$,

(c) $\left.\forall p<\kappa_{v}, y_{v}^{p} \in\right] y_{v}^{p-1} ; a_{v}^{n_{v}-1}[$,

(d) $\lim _{p \rightarrow+\infty} y_{v}^{p}=a_{v}^{n_{v}-1}$.

For every $p \geq 0$, let $A_{v}^{p}=\left[a_{v}^{n_{v}-1} ; x_{v}^{p}\left[\right.\right.$ and $B_{\tau(v)}^{p}=\gamma_{l(v)}\left(A_{v}^{p}\right)=\left[a_{\tau(v)}^{n_{v}-2} ; y_{\tau(v)}^{p}[\right.$.

Lemma 3.5. $\forall v \in V, \forall z \in Z, \forall p \geq 0, F\left(A_{v}^{p}, z\right)=F\left(B_{\tau(v)}^{p}, \gamma_{l(v)}(z)\right)$.

Proof. We start by noting that additivity for contiguous intervals implies that

$$
\forall z \in Z, F(\emptyset, z)=F(\emptyset \sqcup \emptyset, z)=F(\emptyset, z)+F(\emptyset, z) .
$$

Then $F(\emptyset, z)=0$ for every $z \in Z$. If $v$ is a cusp, then $A_{v}^{p}=B_{\tau(v)}^{p}=\emptyset$ for every $p$, as $\tau(v)$ is also a cusp. We can now suppose that $v$ is an inner vertex, and we will prove the equality in that case by induction over $p \geq 0$.

If $p=0, B_{\tau(v)}^{0}=\emptyset=\gamma_{l(v)}\left(A_{v}^{0}\right)$ and the equality holds because $F(\emptyset, z)=0$.

Now suppose that the equality holds for some $p \geq 0$ and for every $z \in Z, v \in V$. Fix $z \in Z$. Since $\gamma_{\sigma^{j-1}(v)} \cdots \gamma_{v}\left(A_{v}^{p+1}\right) \subset I_{\sigma^{j}(v)}$ for every $0 \leq j \leq n_{v}-2$, the inclusion property yields

$$
F\left(\gamma_{\sigma^{j-1}(v)} \ldots \gamma_{v}\left(A_{v}^{p+1}\right), \gamma_{\sigma^{j-1}(v)} \ldots \gamma_{v}(z)\right)=F\left(\gamma_{\sigma^{j}(v)} \ldots \gamma_{v}\left(A_{v}^{p+1}\right), \gamma_{\sigma^{j}}(v) \ldots \gamma_{v}(z)\right)
$$

thus we get by transitivity that

$$
F\left(A_{v}^{p+1}, z\right)=F\left(\gamma_{\sigma^{n_{v}-2}(v)} \cdots \gamma_{v}\left(A_{v}^{p+1}\right), \gamma_{\sigma^{n_{v}-2}(v)} \cdots \gamma_{v}(z)\right)=F\left(g_{v}\left(A_{v}^{p+1}\right), g_{v}(z)\right) \text {. }
$$

This last interval can be split in two

$$
g_{v}\left(A_{v}^{p+1}\right)=g_{v}\left(\left[a_{v}^{n_{v}-1} ; x_{v}^{p+1}[)=\left[a_{l \nu(v)}^{m_{v}-2} ; x_{\nu(v)}^{p}\left[=\left[a_{l \nu(v)}^{m_{v}-2} ; a_{\nu(v)}^{n_{\nu(v)}-1}\left[\sqcup A_{\nu(v)}^{p} .\right.\right.\right.\right.\right.\right.
$$

Denote by $L_{l \nu(v)}$ this first interval; as it is contiguous to $A_{\nu(v)}^{p}$, we get by additivity that

$$
F\left(A_{v}^{p+1}, z\right)=F\left(L_{l \nu(v)}, g_{v}(z)\right)+F\left(A_{\nu(v)}^{p}, g_{v}(z)\right) .
$$

However, since $L_{l \nu(v)} \subset I_{l \nu(v)}$ on one hand, and by induction on the other hand,

$$
F\left(A_{v}^{p+1}, z\right)=F\left(\gamma_{l \nu(v)}\left(L_{l \nu(v)}\right), \gamma_{l \nu(v)} g_{v}(z)\right)+F\left(\gamma_{l \nu(v)}\left(A_{\nu(v)}^{p}\right), \gamma_{l \nu(v)} g_{v}(z)\right) \text {. }
$$

We can now put those two contiguous intervals back together in

$$
\gamma_{l \nu(v)}\left(L_{l \nu(v)}\right) \sqcup \gamma_{l \nu(v)}\left(A_{\nu(v)}^{p}\right)=\gamma_{l \nu(v)} g_{v}\left(A_{v}^{p+1}\right)=\gamma_{\sigma^{n_{v}-1}(v)} g_{v}\left(A_{v}^{p+1}\right)
$$

and in the end we get

$$
F\left(A_{v}^{p+1}, z\right)=F\left(\gamma_{\sigma^{n_{v}-1}(v)} g_{v}\left(A_{v}^{p+1}\right), \gamma_{\sigma^{n_{v}-1}(v)} g_{v}(z)\right) .
$$




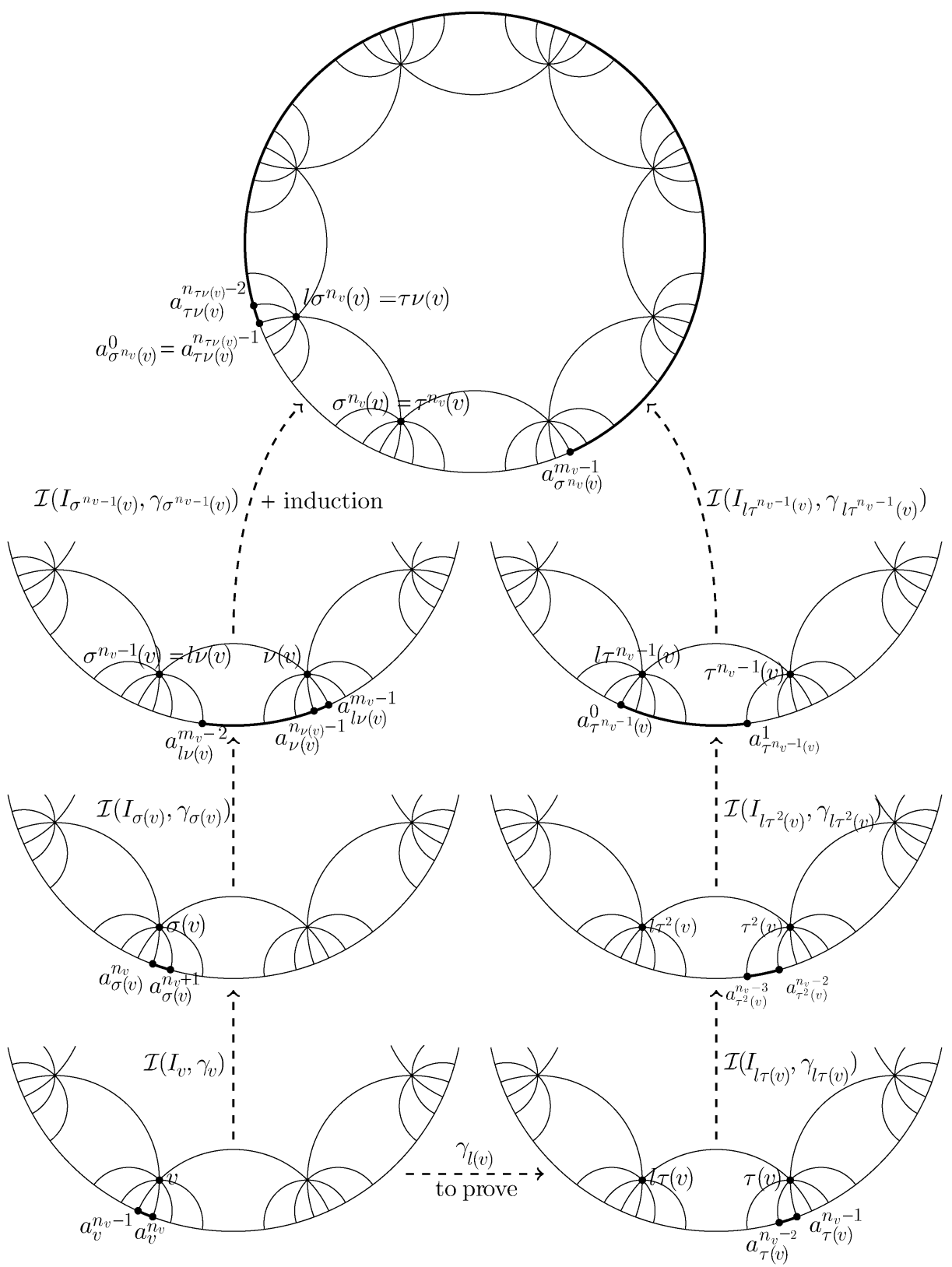

FIGURE 3. Scheme of the core of the induction

On the other hand, $\gamma_{l \tau^{j}(v)} \ldots \gamma_{l \tau(v)}\left(B_{\tau(v)}^{p+1}\right) \subset I_{l \tau^{j+1}(v)}$ for every $0 \leq j \leq n_{v}-2$, so the inclusion property gives that

$$
\begin{aligned}
F\left(\gamma_{l \tau^{j}(v)} \ldots \gamma_{l \tau(v)}\left(B_{\tau(v)}^{p+1}\right)\right. & \left., \gamma_{l \tau^{j}(v)} \ldots \gamma_{l \tau(v)} \gamma_{l(v)}(z)\right) \\
& =F\left(\gamma_{l \tau^{j+1}(v)} \ldots \gamma_{l \tau(v)}\left(B_{\tau(v)}^{p+1}\right), \gamma_{l \tau^{j+1}(v)} \ldots \gamma_{l \tau(v)} \gamma_{l(v)}(z)\right)
\end{aligned}
$$


whose first and last terms are

(4) $F\left(B_{\tau(v)}^{p+1}, \gamma_{l(v)}(z)\right)=F\left(\gamma_{l \tau^{n_{v}-1}(v)} \ldots \gamma_{l \tau(v)}\left(B_{\tau(v)}^{p+1}\right), \gamma_{l \tau^{n_{v}-1}(v)} \ldots \gamma_{l \tau(v)} \gamma_{l(v)}(z)\right)$.

However, since $\gamma_{l \sigma(v)}=\gamma_{v}^{-1}$ for any vertex $v, \gamma_{l \tau^{j}(v)}=\gamma_{l \sigma^{m_{v}-j}(v)}=\gamma_{\sigma^{m_{v}-j-1}(v)}^{-1}$ and

$$
\gamma_{l \tau^{n_{v}-1}(v)} \cdots \gamma_{l(v)}=\gamma_{\sigma^{n_{v}}(v)}^{-1} \ldots \gamma_{\sigma^{m_{v}-1}(v)}^{-1}=\left(\gamma_{\sigma^{m_{v}-1}(v)} \cdots \gamma_{\sigma^{n_{v}}(v)}\right)^{-1} .
$$

The group relation encoded in the $\sigma$-orbit of $v$ gives that $\gamma_{\sigma^{m_{v}-1}(v)} \ldots \gamma_{v}=1$, so

$$
\gamma_{l \tau^{n_{v}-1}(v)} \cdots \gamma_{l(v)}=\left(\gamma_{\sigma^{m_{v}-1}(v)} \cdots \gamma_{\sigma^{n_{v}}(v)}\right)^{-1}=\gamma_{\sigma^{n_{v}-1}(v)} \cdots \gamma_{v}=\gamma_{\sigma^{n_{v}-1}(v)} g_{v} .
$$

Finally,

$$
\begin{aligned}
F\left(\gamma_{l \tau^{n_{v}-1}(v)} \cdots \gamma_{l \tau(v)}\left(B_{\tau(v)}^{p+1}\right),\right. & \left.\gamma_{l \tau^{n_{v}-1}(v)} \cdots \gamma_{l \tau(v)} \gamma_{l(v)}(z)\right) \\
& =F\left(\gamma_{\sigma^{n_{v}-1}(v)} g_{v}\left(A_{v}^{p+1}\right), \gamma_{\sigma^{n_{v}-1}(v)} g_{v}(z)\right)
\end{aligned}
$$

which implies $F\left(A_{v}^{p+1}, z\right)=F\left(B_{\tau(v)}^{p+1}, \gamma_{l(v)}(z)\right)$ thanks to relations (3) and (4).

Lemma 3.6. If $F$ satisfies $\mathcal{I}\left(I_{v}, \gamma_{v}\right)$ for every $v$, then it also satisfies $\mathcal{I}\left(A_{v}, \gamma_{l(v)}\right)$ for every $v$,

$$
\forall v \in V, \forall z \in Z, F\left(A_{v}, z\right)=F\left(B_{\tau(v)}, \gamma_{l(v)}(z)\right) .
$$

Proof. Since $\lim _{p \rightarrow+\infty} x_{v}^{p}=a_{v}^{n_{v}}$ and $\lim _{p \rightarrow+\infty} y_{\tau(v)}^{p}=a_{\tau(v)}^{n_{v}-1}$, the continuity property of $F$ ensures that for every $v \in V$ and $z \in Z$,

$$
F\left(A_{v}, z\right)=\lim _{p \rightarrow+\infty} F\left(A_{v}^{p}, z\right)=\lim _{p \rightarrow+\infty} F\left(B_{\tau(v)}^{p}, \gamma_{l(v)}(z)\right)=F\left(B_{\tau(v)}, \gamma_{l(v)}(z)\right) .
$$

Lemma 3.7. If $F$ satisfies $\mathcal{I}\left(I_{v}, \gamma_{v}\right)$ for every $v$, then it also satisfies $\mathcal{I}\left(\mathbb{S}^{1}, \gamma_{v}\right)$ for every $v$,

$$
\forall v \in V, \forall z \in Z, F\left(\mathbb{S}^{1}, z\right)=F\left(\mathbb{S}^{1}, \gamma_{v}(z)\right) .
$$

Proof. Fix $z \in Z$ and $v \in V$. Recall that $\gamma_{l \sigma(v)}=\gamma_{v}^{-1}$.

We can split $\mathbb{S}^{1}=I_{v} \sqcup{ }^{c} I_{v}$ with ${ }^{c} I_{v}=\gamma_{v}^{-1}\left(I_{l \sigma(v)}\right) \sqcup B_{v} \sqcup A_{r(v)}$, where all those intervals are contiguous. By applying the additivity property, we get that

$$
F\left(\mathbb{S}^{1}, z\right)=F\left(I_{v}, z\right)+F\left(\gamma_{v}^{-1}\left(I_{l \sigma(v)}\right), z\right)+F\left(B_{v}, z\right)+F\left(A_{r(v)}, z\right) .
$$

Likewise, $\mathbb{S}^{1}=I_{l \sigma(v)} \sqcup{ }^{c} I_{l \sigma(v)}$ with ${ }^{c} I_{l \sigma(v)}=\gamma_{v}\left(I_{v}\right) \sqcup B_{l \sigma(v)} \sqcup A_{\sigma(v)}$, so

$$
\begin{aligned}
F\left(\mathbb{S}^{1}, \gamma_{v}(z)\right)=F\left(I_{l \sigma(v)}, \gamma_{v}(z)\right)+F\left(\gamma_{v}\left(I_{v}\right)\right. & \left., \gamma_{v}(z)\right) \\
& +F\left(B_{l \sigma(v)}, \gamma_{v}(z)\right)+F\left(A_{\sigma(v)}, \gamma_{v}(z)\right) .
\end{aligned}
$$

However, since $F$ satisfies $\mathcal{I}\left(I_{l \sigma(v)}, \gamma_{l \sigma(v)}=\gamma_{v}^{-1}\right)$ and $\mathcal{I}\left(I_{v}, \gamma_{v}\right)$,

$$
\begin{aligned}
F\left(I_{l \sigma(v)}, \gamma_{v}(z)\right) & =F\left(\gamma_{v}^{-1}\left(I_{l \sigma(v)}\right), z\right), \\
F\left(\gamma_{v}\left(I_{v}\right), \gamma_{v}(z)\right) & =F\left(I_{v}, z\right) .
\end{aligned}
$$

As for the two last terms, lemma 3.6 gives that

$$
\begin{aligned}
F\left(B_{l \sigma(v)}, \gamma_{v}(z)\right) & =F\left(A_{\sigma l \sigma(v)}, z\right)=F\left(A_{r(v)}, z\right), \\
F\left(A_{\sigma(v)}, \gamma_{v}(z)\right) & =F\left(B_{\tau \sigma(v)}, \gamma_{l \sigma(v)} \gamma_{v}(z)\right)=F\left(B_{v}, z\right) .
\end{aligned}
$$

Hence $F\left(\mathbb{S}^{1}, z\right)=F\left(\mathbb{S}^{1}, \gamma_{v}(z)\right)$. 
We can now finish the proof of the theorem. By lemma 3.7. $F$ satisfies $\mathcal{I}\left(\mathbb{S}^{1}, \gamma_{v}\right)$ for every $v$. Since $\left(\gamma_{v}\right)_{v \in V}$ generates $\Gamma, F$ also satisfies $\mathcal{I}\left(\mathbb{S}^{1}, \gamma\right)$ for every $\gamma \in \Gamma$. The inclusion property finally gives that $F$ satisfies $\mathcal{I}(I, \gamma)$ for every $I \subset \mathbb{S}^{1}, I \in \mathbb{I}$ and $\gamma \in \Gamma$.

\section{Strong orbit-equivalence}

Recall that $T$ acts on each interval $I_{v}$ by an isometry $\gamma_{v} \in \Gamma$. If $x$ is some point of $\mathbb{S}^{1}$ and $k \geq 0$, we define by induction $\gamma_{k}[x] \in \Gamma$ by

$$
\left\{\begin{aligned}
\gamma_{0}[x] & =\mathrm{id} \\
\gamma_{k+1}[x] & =\gamma_{k}[T(x)] \gamma_{v} \text { whenever } x \in I_{v}
\end{aligned}\right.
$$

$\gamma_{k}[x]$ is the element of $\Gamma$ by which $T^{k}$ acts in a neighbourhood of $x$ half-open on the right. It is easy to check that it satisfies the following properties.

Lemma 4.1. If $x \in \mathbb{S}^{1}$ and $p, q \geq 0$ :

(i) $\gamma_{k}[x](x)=T^{k}(x)$;

(ii) $\gamma_{p+q}[x]=\gamma_{p}\left[\gamma_{q}[x](x)\right] \gamma_{q}[x]$.

By applying the invariance theorem of the previous section with a suitable choice for the space $Z$ and the pairing function $F$, we will prove that $T$ actually satisfies a strong orbit-equivalence property, in that the elements of the group $\Gamma$ that can be read along the orbits of $T$ satisfies themselves to the orbit-equivalence property. This result appears as a brief remark in Mor97.

Theorem 4.2. For every $x \in \mathbb{S}^{1}$ and $g \in \Gamma$, there exist $p, q \geq 0$ such that

$$
\gamma_{p}[x]=\gamma_{q}[g(x)] g .
$$

If we specialize this relation in $x$, we get that $T^{p}(x)=T^{q}(g(x))$, which is the very statement of Series' theorem.

Note that this strong orbit-equivalence property is not directly implied by Series' theorem, as the exponents $p$ and $q$ that appear in its statement depend not only on $g$ but also on the base point $x$. The strong orbit-equivalence property is actually equivalent to saying that $p$ and $q$ can be chosen in Series' theorem as being locally constant on a half-open interval that contains $x$.

Lemma 4.3. Let $x \in \mathbb{S}^{1}$ and $h \in \Gamma$. Then, for every $g \in \Gamma$,

$$
\left(\exists p, q \geq 0, \gamma_{p}[x]=\gamma_{q}[h(x)] h\right) \Leftrightarrow\left(\exists p, q \geq 0, \gamma_{p}[g(x)]=\gamma_{q}[h(x)] h g^{-1}\right) .
$$

Proof. We equip $Z=\mathbb{S}^{1} \times \Gamma$ with the left action of $\Gamma$ given by

$$
g \star(x, h)=\left(g(x), h g^{-1}\right) .
$$

For $(x, h) \in Z$ and $I \in \mathcal{I}$, we define

$$
F(I,(x, h))= \begin{cases}1 & \text { if } x \in I \text { and } \exists p, q \geq 0, \gamma_{p}[x]=\gamma_{q}[h(x)] h, \\ 0 & \text { otherwise. }\end{cases}
$$

$F$ clearly satisfies to the inclusion and additivity properties for contiguous intervals. This is also the case for the continuity property because when $\left(b_{n}\right)$ goes to $b$ increasingly, $x \in\left[a ; b\left[\right.\right.$ if and only if there is an $N$ such that $x \in\left[a ; b_{n}\right.$ [ for every $n \geq N$. In order to apply theorem 3.1 we only have to check that

$$
\forall v \in V, F\left(\gamma_{v}\left(I_{v}\right),\left(\gamma_{v}(x), h \gamma_{v}^{-1}\right)\right)=F\left(I_{v},(x, h)\right) .
$$


First suppose that $F\left(I_{v},(x, h)\right)=1$, i.e. $x \in I_{v}$ and there are $p, q \geq 0$ such that $\gamma_{p}[x]=\gamma_{q}[h(x)] h$. Let $y=\gamma_{v}(x)=\gamma_{1}[x](x) \in \gamma_{v}\left(I_{v}\right)$ and $\tilde{h}=\gamma_{v} \star h=h \gamma_{v}^{-1}$, so that $\tilde{h}(y)=h(x)$. Then it comes

$$
\begin{aligned}
\gamma_{p}[y]=\gamma_{p}\left[\gamma_{v}(x)\right] \gamma_{v} \gamma_{v}^{-1} & =\gamma_{p+1}[x] \gamma_{v}^{-1} \\
& =\gamma_{1}\left[\gamma_{p}[x](x)\right] \gamma_{p}[x] \gamma_{v}^{-1} \\
& =\gamma_{1}\left[\gamma_{q}[h(x)] h(x)\right] \gamma_{q}[h(x)] h \gamma_{v}^{-1} \\
& =\gamma_{q+1}[h(x)] h \gamma_{v}^{-1}=\gamma_{q+1}[\tilde{h}(y)] \tilde{h} .
\end{aligned}
$$

Thus we do have $F\left(\gamma_{v}\left(I_{v}\right), \gamma_{v} \star(x, h)\right)=F\left(\gamma_{v}\left(I_{v}\right),(y, \tilde{h})\right)=1$.

Inversely, suppose that $F\left(\gamma_{v}\left(I_{v}\right), \gamma_{v} \star(x, h)\right)=F\left(\gamma_{v}\left(I_{v}\right),(y, \tilde{h})\right)=1$, i.e. $y$ lies in $\gamma_{v}\left(I_{v}\right)$ and there are $p, q \geq 0$ such that $\gamma_{p}[y]=\gamma_{q}[\tilde{h}(y)] \tilde{h}$. Then $x=\gamma_{v}^{-1}(y) \in I_{v}$, $h=\tilde{h} \gamma_{v}, \tilde{h}(y)=h(x)$ and

$$
\gamma_{p+1}[x]=\gamma_{p}\left[\gamma_{1}[x](x)\right] \gamma_{1}[x]=\gamma_{p}[y] \gamma_{v}=\gamma_{q}[\tilde{h}(y)] \tilde{h} \gamma_{v}=\gamma_{q}[h(x)] h .
$$

Hence $F\left(I_{v},(x, h)\right)=1$.

Theorem 3.1 then gives that $F\left(\mathbb{S}^{1},(x, h)\right)=F\left(\mathbb{S}^{1}, g \star(x, h)\right)$ for every $g$ and $(x, h)$. With noticing that $(g \star h)(g(x))=h g^{-1} g(x)=h(x)$, this gives exactly the statement of the lemma.

Now, as we always have $\gamma_{0}[x]=\mathrm{id}=\gamma_{0}[\mathrm{id}(x)] \mathrm{id}$, this lemma shows that there exist $p, q \geq 0$ such that $\gamma_{p}[g(x)]=\gamma_{q}[\operatorname{id}(x)] \operatorname{id} g^{-1}=\gamma_{q}[x] g^{-1}$. If we swap $p$ and $q$, we get the strong orbit-equivalence relation. The proof of the theorem is finished.

The orbit-equivalence property allows us to precisely describe the preimages of periodic points. Let $\operatorname{Per}(T)$ be the set of $T$-periodic points on the circle. If $y \in \operatorname{Per}(T)$, we denote by

$$
\mathcal{T}(y)=\left\{x \in \mathbb{S}^{1} \mid \exists p \geq 0, T^{p}(x)=y\right\}
$$

the set of its preimages. A preimage of a periodic point is called a pre-periodic point.

Proposition 4.4. $y \in \operatorname{Per}(T)$ if and only if $\mathcal{T}(y)=\Gamma y$. In that case, the preimages of $y$ are dense in $\mathbb{S}^{1}$.

Proof. Let $y \in \operatorname{Per}(T)$, and $k>0$ its period. First, if $x \in \mathcal{T}(y)$, then $y=T^{p}(x)$ for some $p$, hence $x=\gamma_{p}[x]^{-1}(y) \in \Gamma y$. Inversely, if $x=\gamma(y)$ with $\gamma \in \Gamma$, then by Series' orbit-equivalence theorem there exist $p, q \geq 0$ such that $T^{p}(\gamma(y))=T^{q}(y)$. Since $p, q$ can be replaced by $p+1, q+1$, we can assume that $k$ divides $q$, in which case $T^{q}(y)=y$. Then $T^{p}(\gamma(y))=y$ and $x=\gamma(y) \in \mathcal{T}(y)$.

Now if $\mathcal{T}(y)=\Gamma y$, then $T(y)=\gamma_{1}[y](y) \in \Gamma y$ is also a preimage of $y$ : there is a $p \geq 0$ such that $T^{p+1}(y)=T^{p}(T(y))=y$. Hence $y \in \operatorname{Per}(T)$.

Finally, $\overline{\Gamma y}=\overline{\mathcal{T}(y)}$ is a closed $\Gamma$-invariant non-empty subset of $\mathbb{S}^{1}$, so it must contain the whole limit set, which is $\mathbb{S}^{1}$ for a group of finite covolume.

\section{Periodic orbits of $T$ And CONJugacy Classes OF HYPERBOLIC ISOMETRIES}

We will now use the strong orbit-equivalence theorem to prove that hyperbolic periodic orbits of $T$ are in bijection with conjugacy classes of primitive hyperbolic isometries of $\Gamma$. 
Recall that $\operatorname{Per}(T)$ is the set of $T$-periodic points. We can equip $\operatorname{Per}(T)$ and $\Gamma$ with two equivalence relations

$$
\begin{aligned}
\forall(x, y) \in \operatorname{Per}(T)^{2}, x & \sim y \Leftrightarrow \exists \gamma \in \Gamma, \gamma(x)=y, \\
\forall(g, h) \in \Gamma^{2}, g & \sim h \Leftrightarrow \exists \gamma \in \Gamma, \gamma g \gamma^{-1}=h .
\end{aligned}
$$

The equivalence classes for $\sim$ in $\operatorname{Per}(T)$ are exactly the periodic orbits of $T$.

Lemma 5.1. Let $x, y \in \operatorname{Per}(T) . x \sim y$ if and only if $x$ and $y$ are on the same periodic orbit.

Proof. Suppose that $x$ and $y$ are $T$-periodic points, and that there exists a $g \in \Gamma$ such that $g(x)=y$. Since $y$ is periodic, $x=g^{-1}(y) \in \Gamma y$ is a pre-periodic point of $y$; hence, by proposition 4.4 there is a $p \geq 0$ such that $T^{p}(x)=y$. As $y$ is a forward image of the periodic point $x$, they are on the same periodic orbit. Inversely, if $x$ and $y$ are on the same periodic orbit, $y=T^{p}(x)=\gamma_{p}[x](x)$ for some $p$, where $\gamma_{p}[x] \in \Gamma$.

We then define

$$
\begin{aligned}
\Phi: \operatorname{Per}(T) & \rightarrow \Gamma, \\
x & \mapsto \gamma_{p(x)}[x] \text { where } p(x)>0 \text { is the period of } x .
\end{aligned}
$$

$\Phi$ is compatible with those relations.

Lemma 5.2. Let $x, y \in \operatorname{Per}(T)$. If $x \sim y$, then $\Phi(x) \sim \Phi(y)$.

Proof. If $x, y \in \operatorname{Per}(T)$ are $\sim$-equivalent, then by lemma 5.1 they are on the same orbit, so they share the same period $k=p(x)=p(y)$ and there exists $p \geq 0$ such that $y=T^{p}(x)$. Denote by $g=\Phi(x)=\gamma_{k}[x]$ and $h=\Phi(y)=\gamma_{k}[y]$. We get that

$$
\gamma_{p}[x] g=\gamma_{p}\left[\gamma_{k}[x](x)\right] \gamma_{k}[x]=\gamma_{p+k}[x]=\gamma_{k}\left[\gamma_{p}[x](x)\right] \gamma_{p}[x]=\gamma_{k}[y] \gamma_{p}[x]=h \gamma_{p}[x] .
$$

Hence $g$ and $h$ are conjugated by $\gamma_{p}[x] \in \Gamma$.

Let $\overline{\operatorname{Per}(T)}$ (respectively, $\bar{\Gamma}$ ) be the set of equivalence classes for the relation $\sim$ in $\operatorname{Per}(T)$ (respectively, $\Gamma$ ). Following lemma 5.1] $\overline{\operatorname{Per}(T)}$ can be identified with the set of periodic orbits of $T$. Lemma 5.2 ensures that $\Phi$ induces a well-defined map $\bar{\Phi}: \overline{\operatorname{Per}(T)} \rightarrow \bar{\Gamma}$.

From all the periodic points of $\operatorname{Per}(T)$, we denote by

$$
\operatorname{Per}(T)^{+}=\left\{x \in \operatorname{Per}(T) \mid \Phi(x)=\gamma_{p(x)}[x] \text { is a hyperbolic isometry of } \Gamma\right\}
$$

the set of hyperbolic periodic points of $T$. Because $T$ is expansive outside from the cusps of the fundamental domain, and that cusps can only be fixed by parabolic isometries, the elements of $\operatorname{Per}(T)^{+}$consist only of repulsive fixed points of hyperbolic isometries.

Since any conjugate of a hyperbolic element is also hyperbolic, lemma 5.2 shows that $\operatorname{Per}(T)^{+}$is stable for the equivalence relation $\sim$ in $\operatorname{Per}(T)$. This implies that the set $\overline{\operatorname{Per}(T)^{+}}$of equivalence classes for $\sim$ in $\operatorname{Per}(T)^{+}$is a well-defined subset of $\overline{\operatorname{Per}(T)}$. It can be identified to the set of hyperbolic periodic orbits of the transformation $T$.

Denote by $\mathcal{H}(\Gamma) \subset \Gamma$ the set of hyperbolic primitive isometries of $\Gamma$. Likewise, since $g \in \Gamma$ is primitive and hyperbolic if and only if $\gamma g \gamma^{-1}$ is primitive and hyperbolic for every $\gamma \in \Gamma$, the equivalence classes for $\sim$ in $\mathcal{H}(\Gamma)$ coincide with the 
conjugacy classes in $\Gamma$. Hence $\overline{\mathcal{H}(\Gamma)}$ is a well-defined subset of $\bar{\Gamma}$ composed of all the conjugacy classes of hyperbolic primitive isometries of $\Gamma$.

We can now state the main theorem of this section.

Theorem 5.3. Suppose that $\Gamma$ is a Fuchsian group of finite covolume that admits an even corners fundamental domain $\mathcal{D}$ that is not a compact triangle, and let $T$ be the associated Bowen-Series transform. Then the map

$$
\begin{aligned}
\bar{\Phi}: \overline{\operatorname{Per}(T)^{+}} & \rightarrow \overline{\mathcal{H}(\Gamma)} \\
\bar{x} & \mapsto \overline{\Phi(x)}
\end{aligned}
$$

is a bijection.

We shall first prove that $\bar{\Phi}$ really maps to conjugacy classes of primitive elements.

Lemma 5.4. If $x \in \operatorname{Per}(T)$, then $\Phi(x)$ is primitive.

Proof. Suppose that $T^{k}(x)=x$ where $k=p(x)>0$ is the (minimal) period of $x$, and that $\Phi(x)=\gamma_{k}[x]=g^{n}$ with $g$ primitive. Because $T$ is eventually expansive, $\gamma_{k}[x]$ cannot be the identity, and neither can $g$. Since $\gamma_{k}[x]$ fixes $x$ and differs from id, $g$ must also fix $x$, and theorem 4.2 implies that there are $p, q \geq 0$ such that $\gamma_{p}[x]=\gamma_{q}[x] g$. Moreover, we must have $p \neq q$ since $g \neq \mathrm{id}$. At the cost of possibly swapping $p$ and $q$, changing $g$ into $g^{-1}$ (which is still primitive) and $n$ into $-n$, we can assume that $p>q$.

Let $r=p-q>0$ and $y=\gamma_{q}[x](x)$. Since we have

$$
\gamma_{r}[y]=\gamma_{p-q}\left[\gamma_{q}[x](x)\right] \gamma_{q}[x] \gamma_{q}[x]^{-1}=\gamma_{p}[x] \gamma_{q}[x]^{-1}=\gamma_{q}[x] g \gamma_{q}[x]^{-1} .
$$

$\gamma_{r}[y]$ is primitive as a conjugate of $g$, and fixes $\gamma_{q}[x](x)=y$. Hence, $y \in \operatorname{Per}(T)$ and $y \sim x$, and lemma 5.1 implies that $x$ and $y$ are on the same $T$-orbit, which means that the period of $y$ is also $k$. As $T^{r}(y)=y, r$ must be a multiple of $k$; but the fact that $\gamma_{r}[y]$ is primitive implies that necessarily $r=k$. Thus,

$$
\gamma_{q}[x] g=\gamma_{r}[y] \gamma_{q}[x]=\gamma_{k}\left[\gamma_{q}[x](x)\right] \gamma_{q}[x]=\gamma_{k+q}[x]=\gamma_{q}\left[\gamma_{k}[x](x)\right] \gamma_{k}[x]=\gamma_{q}[x] \gamma^{n}
$$
and $n=1$.

As $\Phi(x)$ must be hyperbolic when $x \in \operatorname{Per}(T)^{+}$, this proves that $\bar{\Phi}$ sends $\overline{\operatorname{Per}(T)^{+}}$ into $\overline{\mathcal{H}(\Gamma)}$. We shall now prove that $\bar{\Phi}$ is injective on $\overline{\operatorname{Per}(T)^{+}}$.

Lemma 5.5. Suppose that $x, y \in \operatorname{Per}(T)^{+}$are such that $\overline{\Phi(x)}=\overline{\Phi(y)}$. Then $\bar{x}=\bar{y}$.

Proof. Let $g_{1}=\Phi(x)$ and $g_{2}=\Phi(y)$. As $\overline{\Phi(x)}=\overline{\Phi(y)}$, there exists some $h \in \Gamma$ such that $g_{1}=h^{-1} g_{2} h$. However, $x$ is the repulsive fixed point of $g_{1}$, so this conjugacy relation implies that $h(x)$ must be the repulsive fixed point of $g_{2} ; y$ already plays this role, so one must have $y=h(x)$ and then $x \sim y$.

Finally, we only have left to prove that $\bar{\Phi}$ is surjective.

Lemma 5.6. For every $g \in \mathcal{H}(\Gamma)$, there exists $x \in \operatorname{Per}(T)^{+}$such that $\overline{\Phi(x)}=\bar{g}$.

Proof. Let $x$ be the repulsive fixed point of $g$. By theorem 4.2 one can find $p, q \geq 0$ such that $\gamma_{p}[x]=\gamma_{q}[x] g$. Because $g \neq \mathrm{id}, p \neq q$. We shall first prove that $p>q$.

Otherwise, $r=q-p>0$, and if $z=\gamma_{p}[x](x)$ we get

$$
\gamma_{p}[x]=\gamma_{q-p}\left[\gamma_{p}[x](x)\right] \gamma_{p}[x] g=\gamma_{r}[z] \gamma_{p}[x] g
$$

which means that $\gamma_{r}[z]=h g^{-1} h^{-1}$ with $h=\gamma_{p}[x]$. In particular, $\gamma_{r}[z]$ fixes $z$ and is hyperbolic (as a conjugate of the hyperbolic isometry $g^{-1}$ ), so $z \in \operatorname{Per}(T)^{+}$. Then 
$z$ must be the repulsive fixed point of $\gamma_{r}[z]$, and thanks to the conjugacy relation this implies that $h^{-1}(z)=x$ must be the repulsive fixed point of $g^{-1}$, but this contradicts the fact that $x$ is already repulsive for $g$.

We know then that $r=p-q>0$. If $z=\gamma_{q}[x](x)$, we get

$$
\gamma_{q}[x] g=\gamma_{p}[x]=\gamma_{p-q}\left[\gamma_{q}[x](x)\right] \gamma_{q}[x]=\gamma_{r}[z] \gamma_{q}[x],
$$

hence $\gamma_{r}[z]=h g h^{-1}$ with $h=\gamma_{q}[x]$. We now see that $\gamma_{r}[z]$ fixes $z=h(x)$ and is hyperbolic, so $z \in \operatorname{Per}(T)^{+}$. Moreover, the period of $z$ must be $r$ since $\gamma_{r}[z]$ is primitive as a conjugate of $g$ primitive, which means that $\Phi(z)=\gamma_{r}[z] \in \bar{g}$.

\section{Eigendistributions of THE TRANSFER OPERATOR}

As stated in the introduction, the transfer operator $\mathcal{L}_{s}$ associated with $T$ is defined for $s \in \mathbb{C}$ by

$$
\mathcal{L}_{s}: \phi \in E \mapsto\left(y \mapsto \sum_{T(x)=y} \frac{\phi(x)}{\left|T^{\prime}(x)\right|^{s}}\right)
$$

with $E$ being the space of functions

$$
E=\left\{\phi: \mathbb{S}^{1} \rightarrow \mathbb{C} \mid \forall k, \phi_{/ I_{k}} \in \mathcal{C}^{1}\left(I_{k}\right)\right\}
$$

where $\left(I_{k}\right)$ is the finite Markov partition preserved by $T$, and $\mathcal{C}^{1}(I)$ denotes the space of complex-valued functions defined on the interval $I$ that are the restriction of a continuously differentiable function defined on an open neighbourhood of $\bar{I}$. Since the set of all the endpoints of the intervals $I_{k}$ is stable under $T$, and because the action of the isometries on the boundary is smooth, this space is naturally preserved by $\mathcal{L}_{s}$. Note that the set of functions on $\mathbb{S}^{1}$ that are $\mathcal{C}^{1}$ on the neighbourhood of every $\overline{I_{v}}$ (where $\left(I_{v}\right)$ is the partition on which $T$ is defined) is not stable under $\mathcal{L}_{s}$.

In this section, we will study the eigendistributions of this operator for the eigenvalue 1, i.e. the linear functionals $\nu$ acting on $E$ that satisfies

$$
\forall \phi \in E,\left\langle\nu, \mathcal{L}_{s} \phi\right\rangle=\langle\nu, \phi\rangle .
$$

In Pol91, Pollicott has shown that when the quotient $\mathbb{D} / \Gamma$ is compact, the parameters $s \in \mathbb{C}$ such that the eigenspace of $\mathcal{L}_{s}$ for the eigenvalue 1 is non-null are exactly the $s$ for which there is a non-zero solution of $\Delta f=s(1-s) f$ that is $\Gamma$-invariant, where $\Delta$ is the hyperbolic Laplacian in $\mathbb{D}$. The proof uses the fact that $\mathcal{L}_{s}$ can be seen in that case as a trace-class operator acting on the sum of spaces of functions on $\mathbb{S}^{1}$ which are analytic on a fixed neighbourhood of each interval $I_{k}$. The Fredholm determinant of $\mathcal{L}_{s}$ can then be related to the Selberg zeta function of $\Gamma$. This argument no longer holds when $\Gamma$ is cofinite because $T$ may have indifferent fixed points that prevent us from building such neighbourhoods.

First consider the bare hyperbolic space, without any Fuchsian group. We denote by $\mathcal{E}_{\lambda}$ the space of smooth eigenfunctions of the hyperbolic Laplacian for the eigenvalue $\lambda, \mathcal{E}_{\lambda}^{e}$ those of which grow at most exponentially in the hyperbolic radius, and $\mathcal{D}^{\prime}\left(\mathbb{S}^{1}\right)$ the space of distributions over $\mathbb{S}^{1}$. Following Hel81, we can associate to every $f \in \mathcal{E}_{\lambda}$ with $\lambda=s(1-s)$ two linear functionals $\mathcal{D}_{f, s}$ and $\mathcal{D}_{f, 1-s}$ acting on $\mathbb{S}^{1}$ and named the Helgason boundary values of $f$. Moreover, if $f \in \mathcal{E}_{\lambda}^{e}$, then $\mathcal{D}_{f, s}$ and $\mathcal{D}_{f, 1-s}$ are distributions over $\mathbb{S}^{1}$. The stunning point is that we can recover $f$ from its Helgason boundary value by testing it against the Poisson kernel $P^{s}(z, x)$. 
Theorem 6.1 (Helgason).

$$
\begin{aligned}
\mathcal{P}^{s}: \mathcal{D}^{\prime}\left(\mathbb{S}^{1}\right) & \rightarrow \mathcal{E}_{s(1-s)}^{e} \\
\nu & \mapsto z \mapsto\left\langle\nu, P^{s}(z, .)\right\rangle
\end{aligned}
$$

is an isomorphism of Banach spaces, with inverse $f \mapsto \mathcal{D}_{f, s}$.

There are two ways for constructing the Helgason boundary values: the classical approach decomposes $f$ in a sum of spherical harmonics with coefficients $\left(a_{n}\right)_{n \in \mathbb{Z}}$ and define $\left\langle\mathcal{D}_{f, s}, \phi\right\rangle=\sum_{n \in \mathbb{Z}} a_{n} \hat{\phi}(n)$ where $\hat{\phi}(n)$ is the $n$-th Fourier coefficient of $\phi$. However, Otal also noted that this distribution can be written as the limit of the integral of the Poisson bracket $\left\{f, P^{s}\right\}$ on circles with increasing radius, which allows to translate the fact that $f$ is invariant by some isometry $\gamma$ in terms of $\mathcal{D}_{f, s}$.

We now choose some additional notation. $\mathcal{E}_{\lambda}^{b}$ will denote the space of smooth bounded functions of $\mathcal{E}_{\lambda}, \Lambda_{\alpha}$ will represent the space of $\alpha$-Hölder functions over $\left[0 ; 2 \pi\right.$ [ that map 0 to 0 , and $\Lambda_{\alpha}^{1}$ will be the space of linear functionals $\nu$ than can be written as weak derivatives of functions from $\Lambda_{\alpha}$, i.e. such that there exists some $g \in \Lambda_{\alpha}$ for which

$$
\forall \phi \in \mathcal{C}^{1}\left(\mathbb{S}^{1}\right), \tilde{\phi}(t)=\phi\left(e^{i t}\right),\langle\nu, \phi\rangle=\tilde{\phi}(2 \pi) g(2 \pi)-\tilde{\phi}(0) g(0)-\int_{0}^{2 \pi} \tilde{\phi}^{\prime}(t) g(t) d t .
$$

Otal proved in Ota98 that every Helgason boundary value of smooth bounded eigenfunctions with spectral parameter $s$ can be seen as the weak derivative of a function of $\Lambda_{\Re(s)}$.

Theorem 6.2 (Otal). Suppose that $0<\Re(s) \leq 1$. Then

$$
\begin{aligned}
\mathcal{P}^{s}: \Lambda_{\Re(s)}^{1} & \rightarrow \mathcal{E}_{s(1-s)}^{b} \\
\nu & \mapsto z \mapsto\left\langle\nu, P^{s}(z, .)\right\rangle
\end{aligned}
$$

is an isomorphism of Banach spaces, with inverse $f \mapsto \mathcal{D}_{f, s}$.

More precisely, if $\nu$ is the weak derivative of $D \in \Lambda_{\alpha}$ with $\Re(s) \leq \alpha \leq 1$, and if $f=\mathcal{P}^{s}(\nu)$, then

$$
\forall z \in \mathbb{D},|f(z)| \leq C(s)\|D\|_{\alpha} e^{-(\alpha-\Re(s)) d(0, z)}
$$

where $C(s)$ is a constant that only depends on $s$ and $\|D\|_{\alpha}$ is the $\alpha$-Hölder norm of $D$.

As a side note, this theorem's estimate implies that the Helgason boundary value associated with a smooth bounded $\Gamma$-invariant eigenfunction of the hyperbolic Laplacian for the spectral parameter $s, 0<\Re(s)<1$, is the derivative of a continuous function that is exactly $\Re(s)$-Hölder, no more, no less.

Corollary 6.3. Suppose that $0<\Re(s)<\alpha \leq 1$. Let $\nu \in \Lambda_{\alpha}^{1}$. If $\mathcal{P}^{s}(\nu)$ is a smooth bounded $\Gamma$-invariant eigenfunction of the Laplacian for the eigenvalue $s(1-s)$, then $\nu=0$.

Proof. Following Otal's theorem, $f(z)=\mathcal{P}^{s}(\nu)(z)$ uniformly converges to 0 when $|z|$ grows. Since $\Gamma$ is non-elementary, it contains some non-trivial non-elliptic element $\gamma$. Pick any $w \in \mathbb{D}$. Then $f\left(\gamma^{n}(w)\right)=f(w)$ for every $n$, where $\left|\gamma^{n}(w)\right|$ is arbitrarily large. As such, $f(w)=\lim _{n \rightarrow+\infty} f\left(\gamma^{n}(w)\right)=0$. 
We now suppose that $0<\Re(s) \leq 1$. Denote by $D_{f, s}$ the $\Re(s)$-Hölder function associated with $\mathcal{D}_{f, s}$. If $\phi \in \mathcal{C}^{1}\left(\mathbb{S}^{1}\right)$, by identifying $\phi$ with its lift $\tilde{\phi}$, one can write

$$
\left\langle\mathcal{D}_{f, s}, \phi\right\rangle=\phi(2 \pi) D_{f, s}(2 \pi)-\phi(0) D_{f, s}(0)-\int_{0}^{2 \pi} \frac{\partial \phi}{\partial x}(x) D_{f, s}(x) d x .
$$

If $I=\left[a ; b\left[\right.\right.$, this formula allows us to extend $\mathcal{D}_{f, s}$ to $\mathcal{C}^{1}(I)$ by

$$
\forall \phi \in \mathcal{C}^{1}(I),\left\langle\mathcal{D}_{f, s}, \phi \mathbb{1}_{I}\right\rangle=\phi(b) D_{f, s}(b)-\phi(a) D_{f, s}(a)-\int_{a}^{b} \frac{\partial \phi}{\partial x}(x) D_{f, s}(x) d x .
$$

This new definition is compatible with the disjoint union of contiguous intervals, in the sense that:

Proposition 6.4. If $c \in I=\left[a ; b\left[\right.\right.$ and $\phi \in \mathcal{C}^{1}(I)$, then

$$
\left\langle\mathcal{D}_{f, s}, \phi \mathbb{1}_{I}\right\rangle=\left\langle\mathcal{D}_{f, s}, \phi \mathbb{1}_{[a ; c[}\right\rangle+\left\langle\mathcal{D}_{f, s}, \phi \mathbb{1}_{[c ; b[}\right\rangle .
$$

This ensures that we can extend $\mathcal{D}_{f, s}$ to piecewise $\mathcal{C}^{1}$ functions over $\mathbb{S}^{1}$ by linearity. In particular, this distribution can be extended to the space $E$ on which $\mathcal{L}_{s}$ acts.

We now consider a Fuchsian group $\Gamma$ such that $\mathbb{D} / \Gamma$ has finite volume and possesses an even corners fundamental domain $\mathcal{D}$ that is not a compact triangle. Proposition 4 from LT08 tells us how we can express the $\gamma$-invariance of $f$ in terms of its Helgason boundary value.

Proposition 6.5. If $f \in \mathcal{E}_{s(1-s)}^{b}$ satisfies $f \circ \gamma=f$ for some $\gamma \in \Gamma$, then

$$
\forall I \in \mathbb{I}, \forall \gamma \in \Gamma, \forall \phi \in \mathcal{C}^{1}(I),\left\langle\mathcal{D}_{f, s}, \frac{\phi \circ \gamma^{-1}}{\left|\gamma^{\prime} \circ \gamma^{-1}\right|^{s}} \mathbb{1}_{\gamma(I)}\right\rangle=\left\langle\mathcal{D}_{f, s}, \phi \mathbb{1}_{I}\right\rangle .
$$

Denote by $\mathcal{E}_{\lambda}^{b}(\Gamma)$ the space of functions of $\mathcal{E}_{\lambda}^{b}$ that are invariant under the action of $\Gamma$. Any linear functional $\nu \in \Lambda_{\Re(s)}^{1}$ can be extended to $E$ thanks to relation (5)).

The main result of this section states that eigendistributions of the transfer operator for the eigenvalue 1 that lie in $\Lambda_{\Re(s)}^{1}$ are exactly Helgason boundary values of smooth bounded eigenfunctions of the hyperbolic Laplacian on $\mathbb{D} / \Gamma$.

Theorem 6.6. Suppose that $\Gamma$ is a Fuchsian group of finite covolume that admits an even corners fundamental domain $\mathcal{D}$ that is not a compact triangle. $\mathcal{L}_{s}$ is the transfer operator with spectral paramater $s \in \mathbb{C}$ for the Bowen-Series transform associated with $\mathcal{D}$. For every $s \in \mathbb{C}$ such that $0<\Re(s) \leq 1, \nu \mapsto \mathcal{P}^{s}(\nu)$ is an isomorphism between:

- the space of linear functionals $\nu \in \Lambda_{\Re(s)}^{1}$ that satisfy

$$
\forall \phi \in E,\left\langle\nu, \mathcal{L}_{s} \phi\right\rangle=\langle\nu, \phi\rangle,
$$

- the space $\mathcal{E}_{s(1-s)}^{b}(\Gamma)$ of smooth bounded $\Gamma$-invariant eigenfunctions of $\Delta$ for the eigenvalue $s(1-s)$.

The reverse implication is the easiest, and is actually the generalization to the finite covolume case of proposition 7 from [LT08]. Let $f \in \mathcal{E}_{s(1-s)}^{b}(\Gamma)$ and $\nu=\mathcal{D}_{f, s}$. By theorem 6.2, $\nu \in \Lambda_{\Re(s)}^{1}$ and proposition 6.5 gives us that

$$
\forall v \in V, \forall \phi \in \mathcal{C}^{1}\left(I_{v}\right),\left\langle\mathcal{D}_{f, s}, \frac{\phi \circ \gamma_{v}^{-1}}{\left|\gamma_{v}^{\prime} \circ \gamma_{v}^{-1}\right|^{s}} \mathbb{1}_{\gamma_{v}\left(I_{v}\right)}\right\rangle=\left\langle\mathcal{D}_{f, s}, \phi \mathbb{1}_{I_{v}}\right\rangle
$$


Hence,

$$
\begin{aligned}
\left\langle\mathcal{D}_{f, s}, \phi\right\rangle & =\sum_{v \in V}\left\langle\mathcal{D}_{f, s}, \phi \mathbb{1}_{I_{v}}\right\rangle=\sum_{v \in V}\left\langle\mathcal{D}_{f, s}, \frac{\phi \circ \gamma_{v}^{-1}}{\left|\gamma_{v}^{\prime} \circ \gamma_{v}^{-1}\right|^{s}} \mathbb{1}_{\gamma_{v}\left(I_{v}\right)}\right\rangle \\
& =\sum_{v \in V}\left\langle\mathcal{D}_{f, s}, \mathcal{L}_{s}\left(\phi \mathbb{1}_{I_{v}}\right)\right\rangle=\left\langle\mathcal{D}_{f, s}, \mathcal{L}_{s}(\phi)\right\rangle .
\end{aligned}
$$

We will now prove the direct implication with the help of the invariance theorem. Pick $\nu \in \Lambda_{\Re(s)}^{1}$ and denote by $f$ its image by $\mathcal{P}^{s}$. We already know that $f \in \mathcal{E}_{s(1-s)}^{b}$, so we only have to prove that $f$ is $\Gamma$-invariant.

Let $Z=\mathcal{C}^{1}\left(\mathbb{S}^{1}\right)$ equipped with the left action of $\Gamma$ given by

$$
\forall \gamma \in \Gamma, \forall \phi \in Z, \gamma \cdot \phi=\frac{\phi \circ \gamma^{-1}}{\left|\gamma^{\prime} \circ \gamma^{-1}\right|^{s}} .
$$

Note that if $I$ is an interval of $\mathbb{S}^{1}$ and $\phi \in Z$, then $\phi \mathbb{1}_{I} \in \mathcal{C}^{1}(I)$. Then we define

$$
\begin{aligned}
F: \mathbb{I} \times Z & \rightarrow \mathbb{C} \\
(I, \phi) & \mapsto\left\langle\nu, \phi \mathbb{1}_{I}\right\rangle .
\end{aligned}
$$

The condition $\mathcal{I}(I, \gamma)$ translates to

$$
\forall \phi \in Z,\left\langle\nu, \phi \mathbb{1}_{I}\right\rangle=\left\langle\nu, \frac{\phi \circ \gamma^{-1}}{\left|\gamma^{\prime} \circ \gamma^{-1}\right|^{s}} \mathbb{1}_{\gamma(I)}\right\rangle .
$$

Since $\gamma_{v}$ is bijective, there is at most one preimage by $T$ in each interval $I_{v}$, and then

$$
\forall \phi \in Z,\left\langle\nu, \phi \mathbb{1}_{I_{v}}\right\rangle=\left\langle\nu, \mathcal{L}_{s}\left(\phi \mathbb{1}_{I_{v}}\right)\right\rangle=\left\langle\nu, \frac{\phi \circ \gamma_{v}^{-1}}{\left|\gamma_{v}^{\prime} \circ \gamma_{v}^{-1}\right|^{s}} \mathbb{1}_{\gamma_{v}\left(I_{v}\right)}\right\rangle .
$$

This means that $\mathcal{I}\left(I_{v}, \gamma_{v}\right)$ is satisfied for every $v \in V$. Moreover, using the fact that $\nu$ is the weak derivative of a continuous function, it is easy to check that $F$ is continuous and additive for contiguous intervals. We shall now prove that $F$ satisfies the inclusion property. This is ensured by the following lemma.

Lemma 6.7. Let $\nu$ be the weak derivative of a continuous function $g$ defined over $\mathbb{S}^{1}$. Let $I \in \mathbb{I}$ be a half-open interval of $\mathbb{S}^{1}$ and $\gamma \in \Gamma$. Suppose that

$$
\forall \phi \in Z,\left\langle\nu, \frac{\phi \circ \gamma^{-1}}{\left|\gamma^{\prime} \circ \gamma^{-1}\right|^{s}} \mathbb{1}_{\gamma(I)}\right\rangle=\left\langle\nu, \phi \mathbb{1}_{I}\right\rangle
$$

Then, for every $J \in \mathbb{I}, J \subset I$,

$$
\forall \phi \in Z,\left\langle\nu, \frac{\phi \circ \gamma^{-1}}{\left|\gamma^{\prime} \circ \gamma^{-1}\right|^{s}} \mathbb{1}_{\gamma(J)}\right\rangle=\left\langle\nu, \phi \mathbb{1}_{J}\right\rangle
$$

Proof. Note $I=\left[a ; b\left[\right.\right.$. For every $\phi \in \mathcal{C}^{1}\left(\mathbb{S}^{1}\right)$ and $c, d \in I, c<d$, we have on one hand

$$
\left\langle\nu, \phi \mathbb{1}_{[c ; d[}\right\rangle=\phi(d) g(d)-\phi(c) g(c)-\int_{c}^{d} \frac{\partial \phi}{\partial x}(x) g(x) d x
$$

and on the other hand

$$
\begin{aligned}
\left\langle\nu, \frac{\phi \circ \gamma^{-1}}{\left|\gamma^{\prime} \circ \gamma^{-1}\right|^{s}} \mathbb{1}_{\gamma[c ; d[}\right\rangle=\phi(d) \frac{g(\gamma(d))}{\gamma^{\prime}(d)^{s}}- & \phi(c) \frac{g(\gamma(c))}{\gamma^{\prime}(c)^{s}} \\
& -\int_{\gamma(c)}^{\gamma(d)} \frac{\partial}{\partial x}\left(\frac{\phi \circ \gamma^{-1}}{\left(\gamma^{\prime} \circ \gamma^{-1}\right)^{s}}\right)(x) g(x) d x
\end{aligned}
$$


where this last integral can be rewritten as

$$
\begin{aligned}
\int_{\gamma(c)}^{\gamma(d)} \frac{\partial}{\partial x}\left(\frac{\phi \circ \gamma^{-1}}{\left(\gamma^{\prime} \circ \gamma^{-1}\right)^{s}}\right)(x) g(x) d x & =\int_{c}^{d} \gamma^{\prime}(x) \frac{\partial}{\partial x}\left(\frac{\phi \circ \gamma^{-1}}{\left(\gamma^{\prime} \circ \gamma^{-1}\right)^{s}}\right)(\gamma(x)) g(\gamma(x)) d x \\
& =\int_{c}^{d} \frac{\partial}{\partial x}\left(\frac{\phi}{\gamma^{\prime s}}\right)(x) g(\gamma(x)) d x \\
= & \int_{c}^{d} \frac{\partial \phi}{\partial x}(x) \frac{g(\gamma(x))}{\gamma^{\prime}(x)^{s}} d x \\
& +\int_{c}^{d} \phi(x) \frac{\partial}{\partial x}\left(\frac{1}{\gamma^{\prime s}}\right)(x) g(\gamma(x)) d x .
\end{aligned}
$$

Let $\delta(x)=\frac{g(\gamma(x))}{\gamma^{\prime}(x)^{s}}-g(x)$ and $\lambda(x)=\frac{\partial}{\partial x}\left(\frac{1}{\gamma^{\prime}(x)^{s}}\right)(x) g(\gamma(x))$. Subtracting those two relations, we can write

$$
\begin{aligned}
\left\langle\nu, \frac{\phi \circ \gamma^{-1}}{\left|\gamma^{\prime} \circ \gamma^{-1}\right|^{s}} \mathbb{1}_{\gamma[c ; d[}\right\rangle-\left\langle\nu, \phi \mathbb{1}_{[c ; d[}\right\rangle & \\
& =\phi(d) \delta(d)-\phi(c) \delta(c)-\int_{c}^{d} \frac{\partial \phi}{\partial x}(x) \delta(x) d x-\int_{c}^{d} \phi(x) \lambda(x) d x .
\end{aligned}
$$

We will now prove that $\delta$ is continuously differentiable over $\mathbb{R}$. For $c=a$ and $d=b$, one gets that

$$
\phi(b) \delta(b)-\phi(a) \delta(a)-\int_{a}^{b} \frac{\partial \phi}{\partial x}(x) \delta(x) d x=\int_{a}^{b} \phi(x) \lambda(x) d x .
$$

Pick $c \in] a ; b\left[\right.$ and $\epsilon>0$. One can construct an increasing function $\phi_{\epsilon} \in \mathcal{C}^{1}(I)$ such that $\phi_{\epsilon}=0$ on $[a ; c-\epsilon]$ and $\phi_{\epsilon}=1$ on $[c ; b]$. If we specialize the previous relation for this $\phi_{\epsilon}$, we get for $\epsilon$ sufficiently small that

$$
\delta(b)-\int_{c-\epsilon}^{c} \frac{\partial \phi_{\epsilon}}{\partial x}(x) \delta(x) d x=\int_{a}^{b} \phi_{\epsilon}(x) \lambda(x) d x .
$$

For every $x \in[a ; b], \lim _{\epsilon \rightarrow 0} \phi_{\epsilon}(x)=\mathbb{1}_{[c ; b]}(x)$ hence, by Lebesgue's dominated convergence theorem, we get that

$$
\lim _{\epsilon \rightarrow 0} \int_{a}^{b} \phi_{\epsilon}(x) \lambda(x) d x=\int_{c}^{b} \lambda(x) d x .
$$

On the other hand, since $\int_{c-\epsilon}^{c} \frac{\partial \phi_{\epsilon}}{\partial x}(x) d x=\phi_{\epsilon}(c)-\phi_{\epsilon}(c-\epsilon)=1$ and $\frac{\partial \phi_{\epsilon}}{\partial x}(x) \geq 0$,

$$
\begin{aligned}
\left|\int_{c-\epsilon}^{c} \frac{\partial \phi_{\epsilon}}{\partial x}(x) \delta(x) d x-\delta(c)\right| & =\left|\int_{c-\epsilon}^{c} \frac{\partial \phi_{\epsilon}}{\partial x}(x)(\delta(x)-\delta(c)) d x\right| \\
& \leq \int_{c-\epsilon}^{c} \frac{\partial \phi_{\epsilon}}{\partial x}(x)|\delta(x)-\delta(c)| d x .
\end{aligned}
$$

If $\alpha>0$, then since $\delta$ is continuous (because $g$ is continuous) there is $\epsilon_{0}>0$ such that for every $x$ that satisfies $|x-c|<\epsilon_{0},|\delta(x)-\delta(c)|<\alpha$. Now for any $\epsilon<\epsilon_{0}$,

$$
\left|\int_{c-\epsilon}^{c} \frac{\partial \phi_{\epsilon}}{\partial x}(x) \delta(x) d x-\delta(c)\right| \leq \alpha \int_{c-\epsilon}^{c} \frac{\partial \phi_{\epsilon}}{\partial x}(x) d x=\alpha .
$$


Hence, $\lim _{\epsilon \rightarrow 0} \int_{c-\epsilon}^{c} \frac{\partial \phi_{\epsilon}}{\partial x}(x) \delta(x) d x=\delta(c)$. Taking the limit in (타), one finally gets that

$$
\delta(b)-\delta(c)=\int_{c}^{b} \lambda(x) d x .
$$

Thus $\delta$ is $\mathcal{C}^{1}$ over $\bar{I}$ and: $\forall x \in I, \delta^{\prime}(x)=\lambda(x)$.

Now we get back to our previous computation for $c<d$,

$$
\begin{aligned}
\left\langle\nu, \frac{\phi \circ \gamma^{-1}}{\mid \gamma^{\prime} \circ \gamma^{-1 \mid s}}\right. & \left.\mathbb{1}_{\gamma[c ; d[}\right\rangle-\left\langle\nu, \phi \mathbb{1}_{[c ; d[}\right\rangle \\
& =\phi(d) \delta(d)-\phi(c) \delta(c)-\int_{c}^{d} \frac{\partial \phi}{\partial x}(x) \delta(x) d x-\int_{c}^{d} \phi(x) \frac{\partial \delta}{\partial x}(x) d x \\
& =\phi(d) \delta(d)-\phi(c) \delta(c)-\int_{c}^{d} \frac{\partial}{\partial x}(\phi \delta)(x) d x=0
\end{aligned}
$$

which proves the lemma.

$F$ then satisfies all four hypotheses of the invariance theorem, which ensures that $\mathcal{I}\left(\mathbb{S}^{1}, \gamma\right)$ is true for every $\gamma \in \Gamma$,

$$
\forall \gamma \in \Gamma, \forall \phi \in Z,\langle\nu, \phi\rangle=\left\langle\nu, \frac{\phi \circ \gamma^{-1}}{\left|\gamma^{\prime} \circ \gamma^{-1}\right|^{s}}\right\rangle
$$

As $P^{s}(\gamma(z), x)=\frac{P^{s}\left(z, \gamma^{-1}(x)\right)}{\left|\gamma^{\prime} \circ \gamma^{-1}(x)\right|^{s}}$, this means for $f=\mathcal{P}^{s}(\nu)$ that for every $\gamma \in \Gamma$,

$$
f(\gamma(z))=\left\langle\nu, P^{s}(\gamma(z), .)\right\rangle=\left\langle\nu, \frac{P^{s}\left(z, \gamma^{-1}(.)\right)}{\left|\gamma^{\prime} \circ \gamma^{-1}\right|^{s}}\right\rangle=\left\langle\nu, P^{s}(z, .)\right\rangle=f(z)
$$

Hence, $f$ is $\Gamma$-invariant, and this concludes the proof of the theorem.

\section{REFERENCES}

[Bea83] Alan F. Beardon, The Geometry of Discrete Groups, Graduate Texts in Mathematics, vol. 91, Springer Verlag, 1983.

[BS79] Rufus Bowen and Caroline Series, Markov Maps associated with Fuchsians Groups, Publications de l'IHES 50 (1979), 401-418.

[Hel81] Sigurdur Helgason, Topics in Harmonic Analysis on Homogenous Spaces, Progress in Mathematics, vol. 13, Birkhäuser, 1981.

[LT08] A. O. Lopes and Philippe Thieullen, Eigenfunctions of the Laplacian and eigenfunctions of the associated Ruelle operator, Nonlinearity 21 (2008), 2239-2253. MR2439478 (2009m:30081)

[Mor97] Takehiko Morita, Markov systems and transfer operators associated with cofinite Fuchsian groups, Ergodic Theory and Dynamical Systems 17 (1997), 1147-1181. MR1477037 (99d:58137) 
[Ota98] Jean-Pierre Otal, Sur les fonctions propres du Laplacien du disque hyperbolique, Comptes-Rendus de l'Académie des Sciences 327 (1998), no. 1, 161-166. MR. 1645108 (99e:35161)

[Pol91] Mark Pollicott, Some Applications of Thermodynamic Formalism to Manifolds with Constant Negative Curvature, Advances in Mathematics 85 (1991), 161-192.

Département de Mathématiques d’Orsay, Université Paris-Sud 11, 91405 Orsay Cedex, FRANCE

E-mail address: vincent.pit@math.u-psud.fr 